\title{
Grid-based methods for diatomic quantum scattering problems: a finite-element, discrete variable representation in prolate spheroidal coordinates
}

\author{
Liang Tao, ${ }^{1}$ C. W. McCurdy, ${ }^{2,1}$ and T. N. Rescigno ${ }^{1}$ \\ ${ }^{1}$ Lawrence Berkeley National Laboratory, Chemical Sciences, Berkeley, CA 94720 \\ ${ }^{2}$ Departments of Applied Science and Chemistry, University of California, Davis, CA 95616
}

(Dated: November 25, 2008)

\begin{abstract}
We show how to combine finite elements and the discrete variable representation in prolate spheroidal coordinates to develop a grid-based approach for quantum mechanical studies involving diatomic molecular targets. Prolate spheroidal coordinates are a natural choice for diatomic systems and have been used previously in a variety of bound-state applications. The use of exterior complex scaling in the present implementation allows for a transparently simple way of enforcing Coulomb boundary conditions and therefore straightforward application to electronic continuum problems. Illustrative examples involving the bound and continuum states of $\mathrm{H}_{2}^{+}$, as well as the calculation of photoionization cross sections, show that the speed and accuracy of the present approach offer distinct advantages over methods based on single-center expansions.
\end{abstract}

PACS numbers: 34.80.-i,32.80.-t, 31.15.-p

\section{INTRODUCTION}

Understanding and modeling the behavior of molecules subjected to intense, ultrashort laser pulses is theoretically and computationally challenging. Diatomic molecules are of fundamental importance in this regard and provide an important theoretical and experimental testbed for studying a variety of processes, such as multiple ionization by one or more photons, high-order harmonic generation and dissociation [1-3]. An accurate description of these processes relies on the development of theoretical and computational methods capable of providing precise treatments of the electron dynamics.

The development of advanced nonperturbative methods, which involve solving either the time-independent[46] or time-dependent [7-9] Schrödinger equation on a numerical grid, have in recent years provided essentially exact treatments of electron-impact ionization and oneand two-photon double ionization of simple atomic targets. Extension of these methods to simple molecular targets have also begun to appear in the literature [10, 11], but complications arising from the nonspherical nature of the interaction potentials involved adds to the numerical complexity of the implementation. Straightforward extension of atomic close-coupling methods to molecular targets, involving a grid-based treatment of the radial coordinates of the electrons and a single-center expansion of the angular variables in spherical harmonics, is practical for simple molecular targets, but becomes unwieldy with increasing target complexity. We have recently addressed this problem with a hybrid method that combines nuclear-centered analytic basis functions (Gaussians) with numerically-defined grid-based functions [12]. Here we explore an alternative grid-based approach specifically tailored to diatomic targets.

The approach described here is based on the discrete variable representation (DVR) [13] which offers distinct advantages in the representation of local potential energy operators without resorting to the numerical approximations of derivatives that characterize finite-difference methods. As we have previously shown [14], the DVR can be combined with the finite-elements method (FEM), which provides for more flexibility in the design of the numerical grid and increases the sparseness of the Hamiltonian matrix. Another key advantage of using finiteelements is that it allows for the straightforward application of exterior complex scaling(ECS) [4], which simplifies the imposition of asymptotic scattering boundary conditions. In this paper, we show how to develop an exterior-scaled FEM-DVR scheme in prolate spheroidal coordinates, which is optimally suited to the study of diatomic targets.

We begin with the description of a general one-electron diatomic target in prolate spheroidal coordinates in Sec. II. In Sec. III we outline a procedure for constructing a two-dimensional DVR in these coordinates. Section IV discusses an alternative approach involving a DVR in one spheroidal variable coupled with an analytic expansion in the angular spheroidal variable. Section $\mathrm{V}$ gives a number of illustrative examples involving $\mathrm{H}_{2}^{+}$. We conclude with a brief discussion.

\section{PROLATE SPHEROIDAL COORDINATES}

Prolate spheroidal coordinates $(\xi, \eta, \phi)$ are a threedimensional system of coordinates obtained by rotating a two-dimensional elliptic coordinate system about the focal axis of the ellipse. The angle of rotation is defined by $\phi(0 \leq \phi \leq 2 \pi)$. With the foci located at $\pm a$ along the $z$-axis and $r_{1}$ and $r_{2}$ denoting the distances to the two foci, the dimensionless coordinates $(\xi, \eta)$ are defined as

$$
\begin{aligned}
& \xi=\frac{r_{1}+r_{2}}{2 a} \quad(1 \leq \xi \leq \infty) \\
& \eta=\frac{r_{1}-r_{2}}{2 a} \quad(-1 \leq \eta \leq 1),
\end{aligned}
$$


and the back transformation to Cartesian coordinates is

$$
\begin{aligned}
& x=a \sqrt{\left(\xi^{2}-1\right)\left(1-\eta^{2}\right)} \cos \phi \\
& y=a \sqrt{\left(\xi^{2}-1\right)\left(1-\eta^{2}\right)} \sin \phi \\
& z=a \xi \eta .
\end{aligned}
$$

The Laplacian in these coordinates is

$$
\nabla=\frac{1}{a^{2}\left(\xi^{2}-\eta^{2}\right)}\left[\frac{\partial}{\partial \xi}\left(\xi^{2}-1\right) \frac{\partial}{\partial \xi}+\frac{\partial}{\partial \eta}\left(1-\eta^{2}\right) \frac{\partial}{\partial \eta}+\frac{1}{\left(\xi^{2}-1\right)} \frac{\partial^{2}}{\partial \phi^{2}}+\frac{1}{\left(1-\eta^{2}\right)} \frac{\partial^{2}}{\partial \phi^{2}}\right]
$$

and the volume element is

$$
d V=a^{3}\left(\xi^{2}-\eta^{2}\right) d \xi d \eta d \phi .
$$

We will consider the Schrödinger equation for an electron in the field of two fixed nuclei,

$$
\left(-\frac{1}{2} \nabla+\mathcal{V}-E\right) \Psi(\xi, \eta, \phi)=0,
$$

where the Coulomb interaction $V$ between an electron and two nuclei with charges $Z_{1}$ and $Z_{2}$ at the foci is given by

$$
\mathcal{V}(\xi, \eta)=-\frac{Z_{1}}{r_{1}}-\frac{Z_{2}}{r_{2}}=-\frac{\left(Z_{1}+Z_{2}\right) \xi+\left(Z_{2}-Z_{1}\right) \eta}{a\left(\xi^{2}-\eta^{2}\right)} .
$$

We can separate the $\phi$-dependence from $\Psi$ by writing

$$
\Psi(\xi, \eta, \phi)=\sum_{m} \Psi^{m}(\xi, \eta) \frac{e^{i m \phi}}{\sqrt{2 \pi}} \quad m=0, \pm 1, \pm 2 \ldots
$$

to get

$$
\begin{aligned}
& \left(H^{m}(\xi, \eta)-E\right) \Psi(\xi, \eta, \phi)= \\
& \left(-\frac{1}{2 a^{2}\left(\xi^{2}-\eta^{2}\right)}\left[\frac{\partial}{\partial \xi}\left(\xi^{2}-1\right) \frac{\partial}{\partial \xi}+\frac{\partial}{\partial \eta}\left(1-\eta^{2}\right) \frac{\partial}{\partial \eta}-\frac{m^{2}}{\xi^{2}-1}-\frac{m^{2}}{1-\eta^{2}}+2 a\left(Z_{1}+Z_{2}\right) \xi+2 a\left(Z_{2}-Z_{1}\right) \eta\right]\right. \\
& -E) \Psi^{m}(\xi, \eta)=0
\end{aligned}
$$

It is useful at this point to make some remarks about Eq. 8. We note first that when taking matrix elements of the Hamiltonian, the factor $1 /\left(\xi^{2}-\eta^{2}\right)$ is cancelled by the volume element, thus rendering the singularities in the Coulomb interaction at $\xi=1, \eta= \pm 1$ benign. Secondly, we note that the $a$-dependence of the Hamiltonian appears as simple multiplicative factors, which greatly simplifies the construction of the Hamiltonian matrix at different internuclear separations. These two facts highlight the advantage gained by using prolate spheroidal coordinates to study diatomic targets. Another important point to note is that $\Psi^{m}$ is nonanalytic at $\xi=1, \eta= \pm 1$ for odd $m$, behaving as $\left(\xi^{2}-1\right)^{|m| / 2}\left(1-\eta^{2}\right)^{|m| / 2}[3,15]$. Consequently, the numerical implementations we develop for even and odd $m$ will have to be different.

We will now consider two different approaches to solving Eq. (8), the first involving construction of a twodimensional product DVR basis in $\xi$ and $\eta$. The second approach uses an analytic basis expansion for the $\eta$-dependence and a DVR in $\xi$ to solve the resulting coupled equations.

\section{A TWO-DIMENSIONAL DVR}

We begin by expanding the wave function $\Psi^{m}(\xi, \eta)$ in a product basis of DVR functions:

$$
\Psi^{m}(\xi, \eta)=\sum_{i, j}=c_{i j} f_{i}(\xi) g_{j}(\eta)
$$

The variable $\xi$ runs from 1 to some specified maximum value $\xi_{\max }$ at which the grid is truncated. This range is divided into sub-intervals or elements and the DVR functions $\left\{f_{i}(\xi)\right\}$ are constructed from elementary functions $\left\{\chi_{i}(\xi)\right\}$ with compact support. A single "bridging" function associated with each finite-element boundary connects the functions in adjacent elements and provides continuity across the element boundaries [14]. Exterior complex scaling, which scales the $\xi$ variable by a phase factor $e^{i \theta}$ beyond some specified point $\xi_{0}$, is easily implemented with finite elements by simply choosing the point $\xi_{0}$ to coincide with one of the element boundaries. Complex scaling is unnecessary for the variable $\eta[16]$, which runs from -1 to 1 , so we can choose a single element in 
constructing the DVR functions $\left\{g_{j}(\eta)\right\}$; in this case the elementary functions $\chi$ are the same as the DVR functions. Further details about the FEM-DVR constructions can be found in refs. [4] and [14].

Further specification of the basis functions depends on whether $m$ is even or odd for the reasons stated above. We will first outline the case for even $m$ and then discuss the modifictions needed for odd $m$.

\section{A. Even $m$}

For even $m$, the elementary DVR functions are defined as

$$
\chi_{n}(x)=\frac{1}{\sqrt{w_{n}}} \prod_{i \neq n}^{N} \frac{x-x_{i}}{x_{n}-x_{i}},
$$

where $x_{i}$ and $w_{i}$ are the points and associated weights corresponding to some specified $N$-point Gauss quadrature. Matrix elements of all local operators have a simple diagonal representation when evaluated using the underlying quadrature rule:

$$
\begin{aligned}
\int \chi_{n}(x) F(x) \chi_{m}(x) d x & \approx \sum_{k} \chi_{n}\left(x_{k}\right) F\left(x_{k}\right) \chi_{m}\left(x_{k}\right) w_{k} \\
& =F\left(x_{n}\right) \delta_{n, m}
\end{aligned}
$$

which follows from the fact that $\chi_{n}\left(x_{m}\right)=\delta_{n, m} / \sqrt{w_{n}}$.

The kinetic energy and potential matrix elements are simple in this basis. The volume element cancels the factor of $1 /\left(\xi^{2}-\eta^{2}\right)$ that appears in every term in the Hamiltonian, giving:

$$
\begin{aligned}
& \frac{H_{i j, k l}^{m}}{a}=\frac{1}{a} \iint \chi_{i}(\xi) \chi_{j}(\eta) H^{m}(\xi, \eta) \chi_{k}(\xi) \chi_{l}(\eta) d V \\
& =\frac{\delta_{j l}}{2} \int\left(\xi^{2}-1\right) \frac{\partial \chi_{i}}{\partial \xi} \frac{\partial \chi_{k}}{\partial \xi} d \xi+\frac{\delta_{i k}}{2} \int\left(\eta^{2}-1\right) \frac{\partial \chi_{j}}{\partial \eta} \frac{\partial \chi_{l}}{\partial \eta} d \eta \\
& +\frac{\delta_{i k} \delta_{j l}}{2}\left[\frac{m^{2}}{\left(\xi_{i}^{2}-1\right)}+\frac{m^{2}}{\left(1-\eta_{j}^{2}\right)}\right. \\
& \left.-2 a\left(Z_{1}+Z_{2}\right) \xi_{i}-2 a\left(Z_{2}-Z_{1}\right) \eta_{j}\right]
\end{aligned}
$$

where we have used the quadrature rule to evaluate the local centrifugal and potential terms and integration by parts to simplify the derivative parts of the kinetic energy matrix, dropping the surface terms which either vanish at the limits or cancel in an FEM basis when continuity conditions are imposed across the FEM boundaries [14].

We note that the 2D overlap in this basis is

$$
\begin{aligned}
S_{i j, k l} & =\int \chi_{i}(\xi) \chi_{j}(\eta) \chi_{k}(\xi) \chi_{l}(\eta) a^{3}\left(\xi^{2}-\eta^{2}\right) d \xi d \eta \\
& =\delta_{i k} \delta_{j l} a^{3}\left(\xi_{i}^{2}-\eta_{j}^{2}\right) .
\end{aligned}
$$

The overlap cannot be transformed out of the problem by a redefinition of the one-dimensional DVR functions because it depends on how these functions are paired, due to the nonseparability of the volume element. However, the overlap can easily be transformed out of the problem after the Hamiltonian matrix is constructed by using

$$
\hat{H}_{i j, k l}^{m}=\frac{H_{i j, k l}^{m}}{a^{3} \sqrt{\xi_{i}^{2}-\eta_{j}^{2}} \sqrt{\xi_{k}^{2}-\eta_{l}^{2}}} .
$$

We now address the question of boundary conditions and the choice of the underlying Gaussian quadrature for the DVR. The procedure we have used here differs from what was used previously. In our earlier work in which the FEM-DVR was implemented in spherical coordinates, we made the traditional change of variable in the Schrödinger equation to get radial equations whose solutions must vanish at the origin. This boundary condition was enforced by choosing Gauss-Lobatto quadrature [17], which includes the finite-element boundaries as quadrature points, as the underlying quadrature for the DVR and then simply excluding the function associated with the first point from the basis, since all the other DVR functions vanish at that point. In the present case, we follow Esry and Sadeghpour [18] in working with the original Schrödinger equation, making no change of variable to produce radial equations. The FEM-DVR representation of the kinetic energy (Eq. (12)) is Hermitian (or in the case of ECS, complex symmetrc) and the boundary conditions on the wave function at $\xi=1$ and $\eta= \pm 1$ (where the wave function for $m=0$ is finite and zero for other $m$-values) are properly handled by the volume element. So the simplest choice for the DVR is to use a single Gauss-Legendre DVR for the $\eta$ variable, where the endpoints are not quadrature points. For the $\xi$ DVR, we use Gauss-Radau quadrature for the first element, fixing only the right-hand boundary and including all elementary functions in the basis, and Gauss-Lobatto quadrature for the remaining elements. This prescription allows us to construct a proper FEM-DVR and works for all $m$-values.

\section{B. Odd $m$}

For odd $m$ values, the procedure outlined above converges slowly due to the nonanalytic behavior of the wave functions at $\xi,|\eta|=1$. The remedy for these cases is to define the DVR basis functions with appropriate factors of $\left(\xi^{2}-1\right)^{1 / 2}$ and $\left(1-\eta^{2}\right)^{1 / 2}$ built in. For odd $m$, the elementary DVR functions are defined as:

$$
\begin{aligned}
\chi_{n}(\xi) & =\left(\xi^{2}-1\right)^{1 / 2} f_{n}(\xi) \\
f_{n}(\xi) & =\frac{1}{\sqrt{w_{n}}} \frac{1}{\sqrt{\xi_{n}^{2}-1}} \prod_{i \neq n}^{N} \frac{\xi-\xi_{i}}{\xi_{n}-\xi_{i}} \\
\chi_{n}(\eta) & =\left(1-\eta^{2}\right)^{1 / 2} f_{n}(\eta) \\
f_{n}(\eta) & =\frac{1}{\sqrt{w_{n}}} \frac{1}{\sqrt{1-\eta_{n}^{2}}} \prod_{i \neq n}^{N} \frac{\eta-\eta_{i}}{\eta_{n}-\eta_{i}} .
\end{aligned}
$$


The functions are orthonormal under the quadrature rule. For example we have,

$$
\begin{aligned}
\int \chi_{n}(\xi) \chi_{m}(\xi) d \xi & =\int\left(\xi^{2}-1\right) f_{n}(\xi) f_{m}(\xi) d \xi \\
& \approx \sum_{k}\left(\xi_{k}^{2}-1\right) f_{n}\left(\xi_{k}\right) f_{m}\left(\xi_{k}\right) w_{k}
\end{aligned}
$$

and the small error arising from using this quadrature of the overlap integral does not limit the overall accuracy of the method, as is generally the case in DVR applications [17]. From the definitions in Eq. (15) and the discrete orthonormality of the functions $\left\{\chi_{i}\right\}$, we obtain for the Hamiltonian matrix elements:

$$
\begin{aligned}
& \frac{H_{i j, k l}^{m}}{a}=a^{2} \iint \chi_{i}(\xi) \chi_{j}(\eta) H^{m}(\xi, \eta) \chi_{k}(\xi) \chi_{l}(\eta)\left(\xi^{2}-\eta^{2}\right) d \xi d \eta \\
& =\frac{\delta_{j l}}{2} \int\left(\xi^{2}-1\right)\left(\left(\xi^{2}-1\right) \frac{\partial f_{i}}{\partial \xi} \frac{\partial f_{k}}{\partial \xi}+\xi\left(f_{i} \frac{\partial f_{k}}{\partial \xi}+\frac{\partial f_{i}}{\partial \xi} f_{k}\right)\right) d \xi+\frac{\delta_{i k}}{2} \int\left(\eta^{2}-1\right)\left(\left(1-\eta^{2}\right) \frac{\partial f_{j}}{\partial \eta} \frac{\partial f_{l}}{\partial \eta}-\xi\left(f_{j} \frac{\partial f_{l}}{\partial \eta}+\frac{\partial f_{j}}{\partial \eta} f_{l}\right)\right) d \eta \\
& \quad+\frac{\delta_{i k} \delta_{j l}}{2}\left[\xi_{i}^{2}+\eta_{j}^{2}+\frac{m^{2}}{\left(\xi_{i}^{2}-1\right)}+\frac{m^{2}}{\left(1-\eta_{j}^{2}\right)}-2 a\left(Z_{1}+Z_{2}\right) \xi_{i}-2 a\left(Z_{2}-Z_{1}\right) \eta_{j}\right] .
\end{aligned}
$$

The 2D metric can be handled exactly as in the case of even $m$ via Eq. (14).

\section{CLOSE-COUPLED ONE-DIMENSIONAL DVR}

An alternative to the 2D DVR scheme we have examined is to employ an analytic basis expansion for the "angular" $\eta$ variable [19] along with a DVR for the $\xi$ variable. In this close-coupling scheme, the wave function $\Psi^{m}(\xi, \eta)$ is expanded as

$$
\Psi^{m}(\xi, \eta)=\sum_{i=1}^{N} \sum_{\ell=1}^{\ell_{\max }} c_{i \ell} \chi_{i}(\xi) \Theta_{\ell}^{m}(\eta)
$$

where we take $\Theta_{\ell}^{m}(\eta)$ to be the normalized associated Legendre function

$$
\Theta_{\ell}^{m}(\eta)=(-1)^{m}\left[\frac{2 \ell+1}{2} \frac{(\ell-m) !}{(\ell+m) !}\right]^{\frac{1}{2}} P_{\ell}^{m}(\eta)
$$

The Hamiltonian matrix elements in this basis are given as

$$
\begin{aligned}
& \frac{H_{i \ell, j \ell^{\prime}}^{m}}{a}=a^{2} \iint \chi_{i}(\xi) \Theta_{\ell}^{m}(\eta) H^{m}(\xi, \eta) \chi_{j}(\xi) \Theta_{\ell^{\prime}}^{m}(\eta)\left(\xi^{2}-\eta^{2}\right) d \xi d \eta \\
& =\frac{\delta_{\ell \ell^{\prime}}}{2}\left[\int\left(\xi^{2}-1\right) \frac{\partial \chi_{i}}{\partial \xi} \frac{\partial \chi_{j}}{\partial \xi} d \xi+\delta_{i j}\left(\frac{m^{2}}{\left(\xi_{i}^{2}-1\right)}+\ell(\ell+1)\right)\right] \\
& -\delta_{i j} a\left[\delta_{\ell \ell^{\prime}}\left(Z_{1}+Z_{2}\right) \xi_{i}+\left(Z_{2}-Z_{1}\right)\left(\delta_{\ell \ell^{\prime}-1}\left[\frac{(\ell+m+1)(\ell-m+1)}{(2 \ell+1)(2 \ell+3)}\right]^{1 / 2}+\delta_{\ell \ell^{\prime}+1}\left[\frac{(\ell+m)(\ell-m)}{(2 \ell+1)(2 \ell-1)}\right]\right)\right],
\end{aligned}
$$

where the integrals over $\xi$ are performed using the quadrature rule and the $\eta$ integration was simplified by using the generalized Legendre equation,

$$
\left[\frac{\partial}{\partial \eta}\left(1-\eta^{2}\right) \frac{\partial}{\partial \eta}+\ell(\ell+1)-\frac{m^{2}}{\left(1-\eta^{2}\right)}\right] \Theta_{\ell}^{m}(\eta)=0 .
$$

Note that the overlap matrix in this representation is not diagonal in $\ell$ : 


$$
\begin{aligned}
& a^{3} \iint \chi_{i}(\xi) \Theta_{\ell}^{m}(\eta) \chi_{j}(\xi) \Theta_{\ell^{\prime}}^{m}(\eta)\left(\xi^{2}-\eta^{2}\right) d \xi d \eta= \\
& \delta_{i j}\left[\delta_{\ell \ell^{\prime}}\left(\xi_{i}^{2}-\frac{2 \ell^{2}-2 m^{2}+2 \ell-1}{(2 \ell+3)(2 \ell-1)}\right)-\delta_{\ell \ell^{\prime}-2} \frac{1}{(2 \ell+3)}\left(\frac{(\ell+m+1)(\ell-m+1)(\ell+m+2)(\ell-m+2)}{(2 \ell+1)(2 \ell+5)}\right)^{1 / 2}\right. \\
& \left.\quad-\delta_{\ell \ell^{\prime}+2} \frac{1}{(2 \ell+1)}\left(\frac{(\ell+m-1)(\ell-m-1)(\ell+m)(\ell-m)}{(2 \ell-1)(2 \ell+3)}\right)^{1 / 2}\right]
\end{aligned}
$$

\section{ILLUSTRATIVE EXAMPLES}

\section{A. Bound states of $\mathbf{H}_{2}^{+}$}

We have computed the few lowest bound states of $\mathrm{H}_{2}^{+}$at an internuclear separation of 2 bohr using both schemes outlined above with a view toward achieving convergence to machine accuracy in both cases. The results are shown in Table I. The $\xi$ grid runs from 1 to 80 in these calculations and is subdivided into $1 \mathrm{fi-}$ nite element from 1 to 2 and 10 finite elements from 2 to 80 , with 15 th-order DVR in each element. The $2 \mathrm{D}$ DVR calculations are fully converged by using 9th order Gauss-Legendre DVR in $\eta$, while the close-coupling scheme achieves identical results with $\ell_{\max }=8$ in the angular expansion.

\section{B. Continuum states of $\mathbf{H}_{2}^{+}$} tion

The continuum states of $\mathrm{H}_{2}^{+}$are solutions of the equa-

$$
\left(H-\frac{k^{2}}{2}\right) \Psi_{\mathbf{k}}^{(+)}(\xi, \eta, \phi)=0
$$

To apply ECS to solve this equation, we must first convert it into a driven equation for the outgoing wave part of $\Psi^{(+)}$, using the "two-potential" ECS formalism of McCurdy, Horner and Rescigno [20]. To this end, we partition $\Psi^{(+)}$as follows:

$$
\Psi_{\mathbf{k}}^{(+)}(\xi, \eta, \phi)=\Phi_{0}(\xi, \eta, \phi)+\Psi_{s c}(\xi, \eta, \phi),
$$

where the incoming part of the scattering wave function is specified by $\Phi_{0}$ so that $\Psi_{s c}$ is a solution of a driven equation satisfying outgoing boundary conditions,

$$
\left(H-\frac{k^{2}}{2}\right) \Psi_{s c}(\xi, \eta, \phi)=\left(\frac{k^{2}}{2}-H\right) \Phi_{0}(\xi, \eta, \phi) .
$$

Since the long range behavior of the potential is that of a Coulomb interaction of an electron with a positive charge
$Z=Z_{1}+Z_{2}$, an optimum choice for $\Phi_{0}$ is one that coincides, for large $\xi$, with the one-center Coulomb function, $\psi_{c}^{(+)}(\mathbf{k}, \mathbf{r})$, with $Z=Z_{1}+Z_{2}$ and incoming momentum $\mathbf{k}$. That choice ensures that the there are only outgoing waves in $\Psi_{s c}$, and allows us to make contact with the usual partial wave decomposition the scattering or ionization amplitudes. We note that our approach differs from earlier work on $\mathrm{H}_{2}^{+}$continuum states using prolate spheroidal coordinates where the asymptotic behavior was expressed in terms of two-center phaseshifts [15, 2123].

To avoid having to resolve Eq. (23) for each direction of $\mathbf{k}$, we begin with the partial-wave expansion of $\psi_{c}^{(+)}(\mathbf{k}, \mathbf{r})$ in spherical polar coordinates:

$$
\psi_{c}^{(+)}(\mathbf{k}, \mathbf{r})=\left(\frac{2}{\pi}\right)^{1 / 2} \sum_{\ell, m} \frac{i^{\ell} e^{i \eta_{\ell}}}{k r} \phi_{\ell, k}^{(c)}(r) Y_{\ell m}(\hat{\mathbf{r}}) Y_{\ell m}^{*}(\hat{\mathbf{k}})
$$

where $\phi_{\ell, k}^{(c)}$ is the partial-wave Coulomb function and $\eta_{\ell}=\arg \Gamma(\ell+1-i Z / k)$. The above expansion, along with Eqs. (7) and (19), suggests that we define a "partial-wave driving term" $\Phi_{\ell}^{m}$ as,

$$
\begin{aligned}
\Phi_{\ell}^{m} & =g(\xi) \frac{\phi_{\ell, k}^{(c)}(r(\xi, \eta))}{k r(\xi, \eta)} \Theta_{\ell}^{m}(\cos \theta(\xi, \eta)) \frac{e^{i m \phi}}{\sqrt{2 \pi}} \\
& \equiv g(\xi) f_{\ell, m, k}^{(c)}(r(\xi, \eta), \cos \theta(\xi, \eta)) \frac{e^{i m \phi}}{\sqrt{2 \pi}}
\end{aligned}
$$

where $g(\xi)$ is a cutoff function that goes to zero as $\xi \rightarrow 1$, and the mapping between spherical and prolate spheroidal variables is given through

$$
\begin{aligned}
r & =a \sqrt{\xi^{2}+\eta^{2}-1} \\
\cos \theta & =\frac{\xi \eta}{\sqrt{\xi^{2}+\eta^{2}-1}} .
\end{aligned}
$$

Substitution of Eq. (27) into Eq. (25) gives, after some algebra, 
TABLE I: Energies (a.u.) of $\mathrm{H}_{2}^{+}$bound states at $R=2.0$ bohr obtained with close-coupling expansion and with $2 \mathrm{D}$ DVR. In all cases $\xi \in(1,80)$ with 15 th-order DVR.

\begin{tabular}{|c|c|c|c|c|}
\hline & $1 s \sigma_{g}$ & $2 p \sigma_{u}$ & $2 p \pi_{u}$ & $4 p \pi_{u}$ \\
\hline$\ell_{\max }=3$ & & -0. 167533256943 & 0. 071228224071 & 0. 384084709996 \\
\hline$\ell_{\max }=4$ & -0. 602634189177 & & & \\
\hline$\ell_{\max }=5$ & & -0. 167534392117 & 0. 071228180105 & 0. 384084709963 \\
\hline$\ell_{\max }=6$ & -0. 602634214489 & & & \\
\hline$\ell_{\max }=7$ & & -0. 167534392201 & 0. 071228180104 & 0. 384084709963 \\
\hline$\ell_{\max }=8$ & -0. 602634214492 & & & \\
\hline $2 \mathrm{D} \mathrm{DVR}^{a}$ & -0. 602634214492 & -0. 167534392201 & 0. 071228180104 & 0. 384084709963 \\
\hline Reference [3] & -0. 602634214495 & -0. 167534392202 & & \\
\hline
\end{tabular}

${ }^{a}$ DVR order on $\eta \in(-1,1)$ is 9 .

TABLE II: $\mathrm{H}_{2}^{+}$T-matrix elements for $k=1.0$ a.u. in ${ }^{2} \Sigma_{g}$ symmetry at $R=2.0$ bohr. The $2 \mathrm{D}$ DVR and close-coupling schemes gave identical results.

\begin{tabular}{lccr}
\hline \hline & $l_{0}=0$ & $l_{0}=2$ & $l_{0}=4$ \\
\hline$l=0$ & $-0.396361+0.804073 i$ & $0.014887-0.014689 i$ & $0.000239+0.000336 i$ \\
$l=2$ & $0.014891-0.014685 i$ & $0.309380+0.108172 i$ & $0.016510+0.006677 i$ \\
$l=4$ & $0.000239+0.000336 i$ & $0.016513+0.006678 i$ & $0.050174+0.002904 i$ \\
\hline \hline
\end{tabular}

$$
\begin{aligned}
& \left(\frac{k^{2}}{2}-H^{m}\right) \Psi_{s c, \ell}^{m}(\xi, \eta)=\left(\frac{\left(Z_{1}+Z_{2}\right)}{a \sqrt{\xi^{2}+\eta^{2}-1}}-\frac{\left(Z_{1}+Z_{2}\right) \xi+\left(Z_{2}-Z_{1}\right) \eta}{a\left(\xi^{2}-\eta^{2}\right)}\right) g(\xi) f_{\ell, m, k}^{(c)}(r(\xi, \eta), \cos \theta(\xi, \eta)) \\
& -\frac{1}{2 a^{2}\left(\xi^{2}-\eta^{2}\right)}\left[f_{\ell, m, k}^{(c)}(r(\xi, \eta), \cos \theta(\xi, \eta))\left(\frac{\partial}{\partial \xi}\left(\xi^{2}-1\right) \frac{\partial}{\partial \xi}\right) g(\xi)+2\left(\xi^{2}-1\right) \frac{\partial g(\xi)}{\partial \xi} \frac{\partial f_{\ell, m, k}^{(c)}(r(\xi, \eta), \cos \theta(\xi, \eta))}{\partial \xi}\right]
\end{aligned}
$$

where we have again eliminated the $\phi$ variable from consideration by working with a specific $m$-value, which is a good quantum number. The function $g(\xi)$ is needed to cut off the right-hand side of Eq. (29) near the onecenter singularity at $r=0$ (i.e., at $\xi=1, \eta=0$ in the one-center Coulomb term $\left.\left(Z_{1}+Z_{2}\right) /\left(a \sqrt{\xi^{2}+\eta^{2}-1}\right)\right)$, which is not removed by the use of prolate spheroidal coordinates. From the partial wave-solutions $\Psi_{s c, \ell}^{m}$, we can construct the full solution $\Psi_{s c}$ for any $\mathbf{k}$ :

$\Psi_{s c}(\xi, \eta, \phi)=\left(\frac{2}{\pi}\right)^{1 / 2} \sum_{m} \sum_{\ell=|m|}^{\infty} i^{\ell} e^{i \eta_{\ell}} \Psi_{s c, \ell}^{m}(\xi, \eta) e^{i m \phi} Y_{\ell m}^{*}(\hat{\mathbf{k}})$

It is important to note the the number of $\ell$-values required for convergence is the number needed to ade- quately represent the one-center atomic Coulomb function and should not be confused with the number of angular momentum terms that would be needed in a singlecenter expansion of the wave function in spherical coordinates.

Equation (29) can be solved using either the 2D DVR scheme by expanding $\Psi_{s c, \ell}^{m}(\xi, \eta)$ as in Eq. (9) or by using a close-coupling expansion as in Eq. (18). In either case, we use exterior complex scaling in the $\xi$-variable to inforce the proper outgoing-wave boundary conditions on the scattered wave. The cutoff function $g(\xi)$ was chosen to be a polynomial defined on an interval $\xi \in\left(\xi_{1}, \xi_{2}\right)$ with $g\left(\xi_{1}\right)=0, g\left(\xi_{2}\right)=1$ and zero first and second derivatives at $\xi_{1}$ and $\xi_{2}$. The explicit functional form we used was 
TABLE III: As in Table II, but for ${ }^{2} \Sigma_{u}$ symmetry.

\begin{tabular}{lccr}
\hline \hline & $l_{0}=1$ & $l_{0}=3$ & $l_{0}=5$ \\
\hline$l=1$ & $0.327633+0.123223 i$ & $0.023568+0.011786 i$ & $0.000026+0.000332 i$ \\
$l=3$ & $0.023572+0.011786 i$ & $0.104822+0.011953 i$ & $0.011202+0.001528 i$ \\
$l=5$ & $0.000026+0.000332 i$ & $0.011203+0.001527 i$ & $0.029378+0.001025 i$ \\
\hline \hline
\end{tabular}

TABLE IV: As in Table II, but for ${ }^{2} \Pi_{g}$ symmetry.

\begin{tabular}{lccr}
\hline \hline & $l_{0}=2$ & $l_{0}=4$ & $l_{0}=6$ \\
\hline$l=2$ & $-0.403016+0.204472 i$ & $0.014297-0.006027 i$ & $0.000096+0.000134 i$ \\
$l=4$ & $0.014295-0.006023 i$ & $0.069823+0.005253 i$ & $0.010399+0.001006 i$ \\
$l=6$ & $0.000096+0.000134 i$ & $0.010399+0.001005 i$ & $0.026248+0.000830 i$ \\
\hline \hline
\end{tabular}

$$
\begin{aligned}
g(x) & =\left\{\begin{array}{l}
0, \quad x \leq 0 \\
\frac{1}{2}(m+2)(m+1) x^{m+3}-(m+3)(m+1) x^{m+2}+\left(1+\frac{(m+1)(m+4)}{2}\right) x^{m+1}, \quad 0 \leq x \leq 1 \\
1, \quad x \geq 1
\end{array}\right. \\
x & =\frac{\xi-\xi_{1}}{\xi_{2}-\xi_{1}}
\end{aligned}
$$

The results were found to be insensitive to values of $m$ between 5 and 10 .

Convergence was tested by computing $T$-matrix elements for the $\mathrm{H}_{2}^{+}$continuum functions using the 2D DVR and close-coupling schemes. The $T$-matrix is defined by the asymptotic behavior of $\Psi_{s c, \ell}^{m}$ expressed in spherical coordinates:

$$
\begin{array}{r}
r \Psi_{s c, \ell_{0}}^{m}(\mathbf{r}(\xi, \eta)) \underset{r \rightarrow \infty}{\sim} \sum_{l} \Theta_{l}^{m}(\cos \theta) T_{\ell_{0}, \ell}^{m} \\
\times e^{i\left[k r+(Z / k) \ln 2 k r-\pi l / 2+\eta_{l}(k)\right]}
\end{array}
$$

The $T_{\ell^{0}, \ell^{-m a t r i x}}^{m}$ elements can therefore be obtained by examining the asymptotic behavior of the function obtained by projecting $\Theta_{\ell}^{m}(\cos \theta)$ from $r \Psi_{s c, \ell_{0}}^{m}(\mathbf{r}(\xi, \eta))$. Tmatrix results in ${ }^{2} \Sigma_{g}^{+},{ }^{2} \Sigma_{u}^{+},{ }^{2} \Pi_{g}$ and ${ }^{2} \Pi_{u}$ are given in Tables II-V, respectively. The tabulated results were all obtained with a finite-element $\xi$ grid, running from 1 to 80, which was subdivided into 1 element from 1 to 2 and 10 elements from 2 to 80 with 15 th-order DVR in each element. The 8th element, beginning at $\xi=50$, was complexscaled. For the 2D DVR calculations, we used a single element for $\eta$ on $(-1,1)$ and 13th order Gauss-Legendre DVR, while the close-coupling results were obtained using $\ell_{\max }=8$. The $T$-matrix elements obtained using the two different methods were identical to the number of places given in Tables II-V. Note that the $T$-matrix for this problem should be complex-symmetric. This property is not imposed, but rather can be taken as a measure of numerical convergence. The small asymmetry that is seen in several $T$-matrix elements can be traced to trun- cation of the $\xi$-grid and not to any deficiencies in the treatment of the $\eta$ degree of freedom, since the 2D DVR results are identical to the close-coupling results.

\section{C. $\mathbf{H}_{2}^{+}$photoionization cross sections}

The final example we consider is photoionization of $\mathrm{H}_{2}^{+}$. The photoionization amplitude for a fixed-in-space molecular orientation is defined by

$$
\left\langle\Psi_{\mathbf{k}}^{(-)}|\epsilon \cdot \boldsymbol{\mu}| \Psi_{0}\right\rangle
$$

and the corresponding differential cross section is

$$
\begin{aligned}
\frac{d \sigma(\mathbf{k}, \boldsymbol{\epsilon})}{d \Omega} & =\frac{4 \pi^{2} \omega k}{c}\left|\left\langle\Psi^{(-)}(\mathbf{k}, \mathbf{r})|\boldsymbol{\epsilon} \cdot \mathbf{r}| \Psi_{0}(\mathbf{r})\right\rangle\right|^{2}(\text { length) } \\
& =\frac{4 \pi^{2} k}{\omega c}\left|\left\langle\Psi^{(-)}(\mathbf{k}, \mathbf{r})|\boldsymbol{\epsilon} \cdot \nabla| \Psi_{0}(\mathbf{r})\right\rangle\right|^{2} \text { (velocity) }
\end{aligned}
$$

where $\mathbf{k}$ is the outgoing electron momentum, $\Psi_{0}(\mathbf{r})$ is the initial state with energy $E_{0}, \epsilon$ defines the polarization direction for a photon with energy $\omega, \boldsymbol{\mu}$ is the dipole operator and the final state $\Psi_{\mathbf{k}}^{(-)}$is related to the function in Eq. (24) by

$$
\Psi_{\mathbf{k}}^{(-)}=\left[\Psi_{-\mathbf{k}}^{(+)}\right]^{*}
$$

The photoionization amplitude can be computed from the solution of the perturbative first-order equation

$$
\left[E_{0}+\omega-H\right] \Phi_{s c}=(\boldsymbol{\epsilon} \cdot \boldsymbol{\mu}) \Psi_{0}
$$


TABLE V: As in Table II, but for ${ }^{2} \Pi_{u}$ symmetry.

\begin{tabular}{lccr}
\hline \hline & $l_{0}=1$ & $l_{0}=3$ & $l_{0}=5$ \\
\hline$l=1$ & $0.051511+0.002874 i$ & $0.014503+0.001360 i$ & $0.000042+0.000112 i$ \\
$l=3$ & $0.014504+0.001360 i$ & $0.041746+0.002015 i$ & $0.007459+0.000446 i$ \\
$l=5$ & $0.000042+0.000112 i$ & $0.007459+0.000446 i$ & $0.017760+0.000390 i$ \\
\hline \hline
\end{tabular}

by writing

$$
\begin{aligned}
& \left\langle\Psi_{\mathbf{k}}^{(-)}|\boldsymbol{\epsilon} \cdot \boldsymbol{\mu}| \Psi_{0}\right\rangle= \\
& \left\langle\Psi_{\mathbf{k}}^{(-)}\left|\left(E_{0}+\omega-H\right)\left(E_{0}+\omega-H+i \epsilon\right)^{-1} \boldsymbol{\epsilon} \cdot \boldsymbol{\mu}\right| \Psi_{0}(\mathbf{r})\right\rangle \\
& =\left\langle\Psi_{\mathbf{k}}^{(-)}\left|E_{0}+\omega-H\right| \Phi_{s c}\right\rangle .
\end{aligned}
$$

Equation (36) can be further simplified by using Green's theorem to convert the amplitude to a surface integral, as we will show below.
For this final example, we carried out calculations in both the length and velocity gauges using only the closecoupling scheme. The dipole operator $\boldsymbol{\mu}$ in the length gauge is simply the displacement operator $\mathbf{r}$ whose components are expressed in prolate spheroidal coordinates in Eq. (2). For the velocity gauge, we need to express the Cartesian components of the gradient operator $\boldsymbol{\nabla}$ in prolate spheroidal coordinates, which is accomplished in a straightforward manner using the chain rule,

$$
\begin{aligned}
\frac{d}{d x} & =\frac{d \xi}{d x} \frac{d}{d \xi}+\frac{d \eta}{d x} \frac{d}{d \eta}+\frac{d \phi}{d x} \frac{d}{d \phi}=\frac{2 \sqrt{\left(\xi^{2}-1\right)\left(1-\eta^{2}\right)} \cos \phi}{R\left(\xi^{2}-\eta^{2}\right)}\left[\xi \frac{d}{d \xi}-\eta \frac{d}{d \eta}\right]-\frac{2 \sin \phi}{R \sqrt{\left(\xi^{2}-1\right)\left(1-\eta^{2}\right)}} \frac{d}{d \phi} \\
\frac{d}{d y} & =\frac{d \xi}{d y} \frac{d}{d \xi}+\frac{d \eta}{d y} \frac{d}{d \eta}+\frac{d \phi}{d y} \frac{d}{d \phi}=\frac{2 \sqrt{\left(\xi^{2}-1\right)\left(1-\eta^{2}\right)} \sin \phi}{R\left(\xi^{2}-\eta^{2}\right)}\left[\xi \frac{d}{d \xi}-\eta \frac{d}{d \eta}\right]+\frac{2 \cos \phi}{R \sqrt{\left(\xi^{2}-1\right)\left(1-\eta^{2}\right)}} \frac{d}{d \phi} \\
\frac{d}{d z} & =\frac{d \xi}{d z} \frac{d}{d \xi}+\frac{d \eta}{d z} \frac{d}{d \eta}+\frac{d \phi}{d z} \frac{d}{d \phi}=\frac{2}{R\left(\xi^{2}-\eta^{2}\right)}\left[\eta\left(\xi^{2}-1\right) \frac{d}{d \xi}+\xi\left(1-\eta^{2}\right) \frac{d}{d \eta}\right] .
\end{aligned}
$$

The factor $\sqrt{\left(\xi^{2}-1\right)\left(1-\eta^{2}\right)}$ that appears in the formulas for $\frac{d}{d x}$ and $\frac{d}{d y}$ might appear to pose a problem for numerical quadrature. But the operators $\frac{d}{d x}$ and $\frac{d}{d y}$ only connect functions with even and odd $m$ values, so non-zero matrix elements of these operators have only one function with odd $m$. But for odd $m$, the elementary DVR functions are defined with this factor built into their definition (see Eq. (15), so the required matrix elements can be accurately evaluated using the underlying Gauss quadrature rule.

Having solved the driven equations for $\Psi^{(+)}$and $\Phi_{s c}$, the photionization amplitude is evaluated using Eq. (36). The required volume integral in spheroidal coordinates can be converted, using Green's theorem, into an integral over a surface of constant $\xi_{0}$ :

$$
\begin{aligned}
&\left\langle\Psi_{\mathbf{k}}^{(-)}\left|E_{0}+\omega-H\right| \Phi_{s c}\right\rangle= \\
&=\frac{1}{2} \iint {\left[\Psi_{\mathbf{k}}^{(-) *} \nabla \Phi_{s c}-\Phi_{s c} \nabla \Psi_{\mathbf{k}}^{(-) *}\right] \cdot d \mathbf{S} } \\
&=\frac{a}{2}\left(\xi_{0}^{2}-1\right) \iint\left[\left.\Psi_{\mathbf{k}}^{(-) *}\left(\xi_{0}, \eta, \phi\right) \frac{d}{d \xi} \Phi_{s c}(\xi, \eta, \phi)\right|_{\xi=\xi_{0}}\right. \\
&\left.\quad-\left.\Phi_{s c}\left(\xi_{0}, \eta, \phi\right) \frac{d}{d \xi} \Psi_{\mathbf{k}}^{(-) *}(\xi, \eta, \phi)\right|_{\xi=\xi_{0}}\right] d \eta d \phi
\end{aligned}
$$

The integral over $\phi$ in Eq. (38) is trivial, giving $\delta_{m, m^{\prime}}$ when we sum over the $m=0, \pm 1$ components of $\Phi_{s c}$ and $\Psi_{\mathbf{k}}^{(-)}$allowed by dipole selection rules. The close coupling scheme expresses the $\eta$ dependence of $\Phi_{s c}^{m}(\xi, \eta)$ and $\Psi_{s c, \ell}^{m}(\xi, \eta)$ (Eq. (29)) as an expansion in the functions $\Theta_{\ell}^{m}(\eta)$ (Eq. (19)). If we express the reference atomic Coulomb functions in this same basis by projecting $\Theta_{\ell}^{m}(\eta)$ onto $\phi_{\ell, k}^{(c)}(r(\xi, \eta)) \Theta_{\ell}^{m}(\cos \theta(\xi, \eta))$, then the integral over $\eta$ in Eq. (38) also collapses due to the orthonormality of the functions $\Theta_{\ell}^{m}(\eta)$. The final result can then be expressed as a sum of Wronskian terms evaluated on the surface $\xi=\xi_{0}$.

The photoionization calculations were carried out using the close-coupling scheme, using the same basis set parameters that were employed in computing the $\mathrm{H}_{2}^{+}$continuum functions. Identical results were obtained in the length and velocity gauges. For the total cross section, we can use the expression,

$$
\sigma_{\text {tot }}=\frac{4 \pi \omega}{c} \operatorname{Im}\left\langle\Psi_{0}|\epsilon \cdot \mathbf{r}| \Phi_{s c}\right\rangle
$$

The total photoionization cross sections for polarization parallel and perpendicular to the molecular axis are plotted in Figs. 1 and 2, respectively. The agreement with 


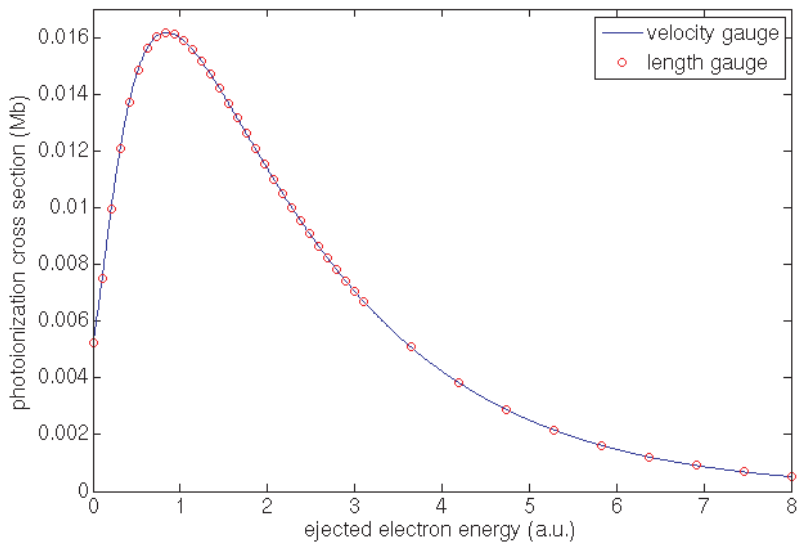

FIG. 1: (Color online) $\mathrm{H}_{2}^{+}$total photoionization cross section, in megabarns, at $\mathrm{R}=2.0 \mathrm{bohr}$ for polarization parallel to the molecular axis. Solid curve: velocity gauge results; Points: length gauge results. $1 \mathrm{Mb}=10^{-18} \mathrm{~cm}^{2}$.

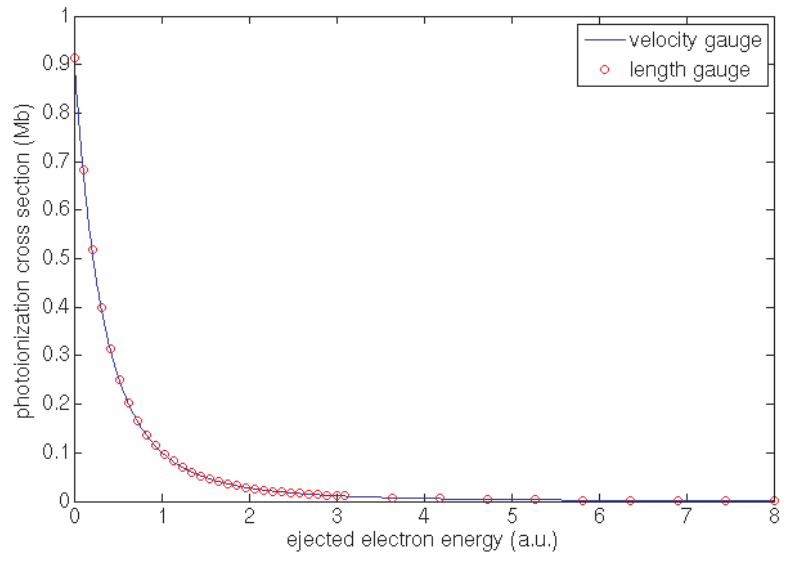

FIG. 2: (Color online) $\mathrm{H}_{2}^{+}$total photoionization cross section at $\mathrm{R}=2.0 \mathrm{bohr}$ for polarization perpendicular to the molecular axis. Solid curve: velocity gauge results; Points: length gauge results.

the accurate numerical results of Bates and Opik [24] is essentially perfect.

The differential photoionization cross sections at $10 \mathrm{eV}$ photoelectron energy are shown in Fig. 3 for four different orientations of the molecule with respect to the direction of polarization. These results are also in good agreement with other recent accurate calculations [25, 26].

\section{DISCUSSION}

Prolate spheroidal coordinates are a natural choice for carrying out quantum mechanical scattering studies involving diatomic targets. We have shown how to develop
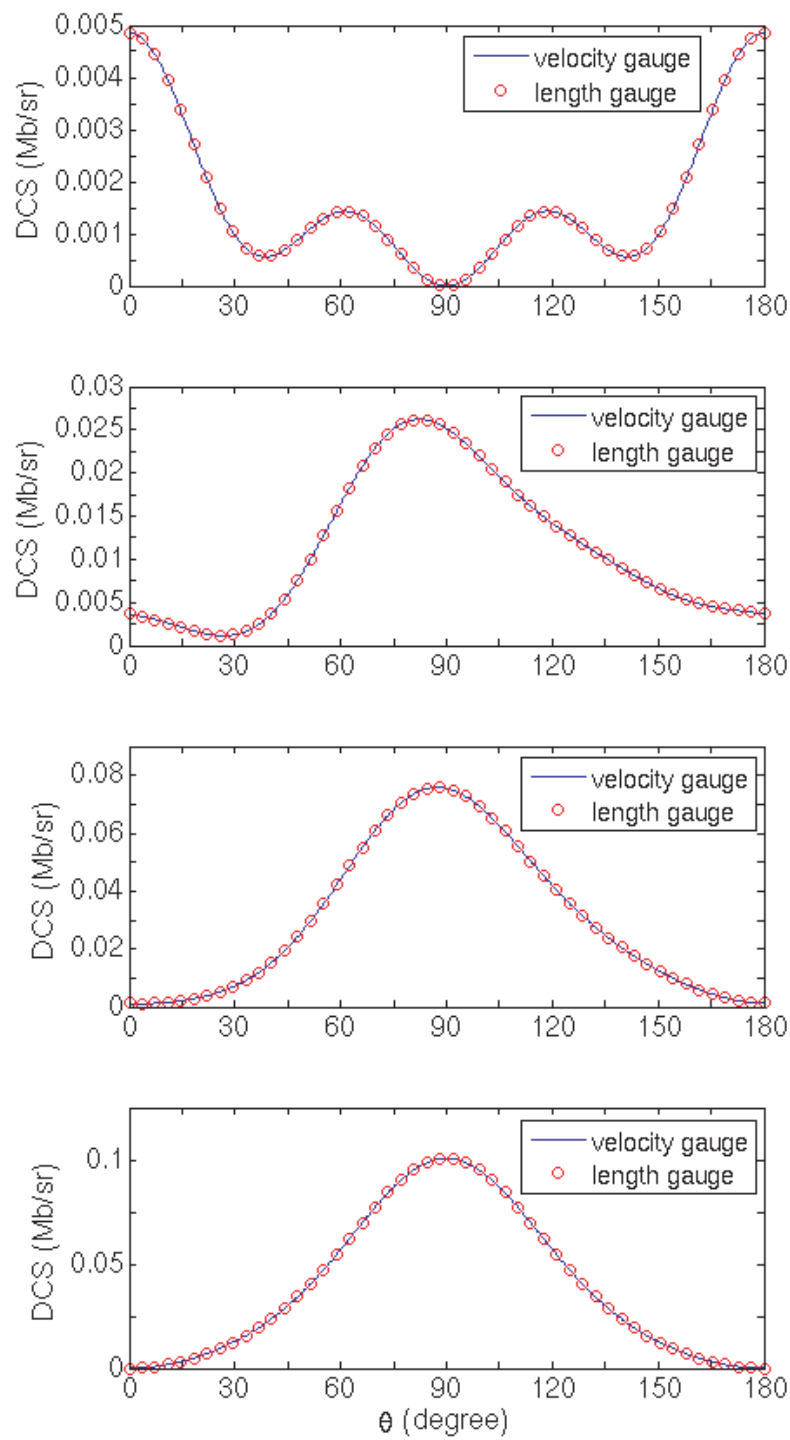

FIG. 3: (Color online) Body-frame differential photoionization cross sections for $\mathrm{H}_{2}^{+}$at $\mathrm{R}=2.0 \mathrm{bohr}$ for four different angles between the molecular axis and the polarization vector. Polarization angles are (top to bottom): $0^{\circ}, 30^{\circ}, 60^{\circ}$, $90^{\circ}$.

a finite-element, discrete variable representation in these coordinates that can be implemented in conjunction with exterior complex scaling. The Hamiltonian dependence on internuclear distance is transparently simple in these coordinates, requiring only a simple scaling of the kinetic energy and electron-nuclear Coulomb interaction. We have demonstrated with several illustrative calculations involving bound and continuum states of $\mathrm{H}_{2}^{+}$that fully converged results can be obtained with modest ex- 
pansions, using either a two-dimensional DVR in $\xi, \eta$ or DVR in $\xi$ along with a close-coupling expansion in $\eta$ using an analytic expansion basis.

While we have focused here on the construction of a one-electron basis for diatomics in prolate spheroidal coordinates, for applications to many-electron diatomic targets the treatment of electron-electron repulsion in these coordinates is also required. The expansion of $1 / r_{12}$ in prolate spheroidal coordinates is in fact well known [2730]. This expansion can also be used in connection with the present FEM DVR scheme. That topic will be the subject of a future study.

\section{Acknowledgments}

The authors have benefited from stimulating discussions with Professor Brett Esry on the treatment of boundary conditions with the DVR. This work was performed under the auspices of the US Department of Energy by the University of California Lawrence Berkeley National Laboratory under Contract DE-AC02$05 \mathrm{CH} 11231$ and was supported by the U.S. DOE Office of Basic Energy Sciences, Division of Chemical Sciences. CWM acknowledges support from the NSF (PHY0604628).
[1] J. H. Posthumus, Rep. Prog. Phys. 67, 623 (2004).

[2] D. A. Telnov and Shih-I Chu, Phys. Rev. A 71, 013408 (2005).

[3] D. A. Telnov and Shih-I Chu, Phys. Rev. A 76, 043412 (2007).

[4] C. W. McCurdy, M. Baertschy, and T. N. Rescigno, J. Phys. B 37, R137 (2004).

[5] D. A. Horner, F. Morales, T. N. Rescigno, F. Martín, and C. W. McCurdy, Phys. Rev. A 76, 030701(R) (2007).

[6] L. Malegat, P. Selles, and A. K. Kazansky, Phys. Rev. A 60, 3667 (1999).

[7] M. S. Pindzola, et al., J. Phys. B 40, R39 (2007).

[8] A. Palacios, T. N. Rescigno, and C. W. McCurdy, Phys. Rev. A 77, 032716 (2008).

[9] J. Feist, S. Nagele, R. Pazourek, E. Persson, B. I. Schneider, L. A. Collins, and J. Burgdorfer, Phys. Rev. A. 77, 043420 (2008).

[10] D. A. Horner, W. Vanroose, T. N. Rescigno, F. Martín, and C. W. McCurdy, Phys. Rev. Lett. 98, 073001 (2007).

[11] J. Colgan, M. S. Pindzola, and F. Robicheaux, Phys. Rev. Lett. 98, 153001 (2007).

[12] F. L. Yip, C. W. McCurdy, and T. N. Rescigno, Phys. Rev. A 78, 023405 (2008).

[13] J. V. Lill, G. A. Parker, and J. C. Light, Chem. Phys. Lett. 89, 483 (1982).

[14] T. N. Rescigno and C. W. McCurdy, Phys. Rev. A 62, 032706 (2000).

[15] L. I. Ponomarev and L. N. Somov, J. Comp. Phys. 20, 183 (1976).
[16] P. Froelich, K. Szalewicz, B. Jeziorski, W. Kolos, and H. J. Monkhorst, J. Phys. B 20, 6173 (1987).

[17] D. E. Manolopoulos and R. E. Wyatt, Chem. Phys. Lett. 152, 23 (1988).

[18] B. D. Esry and H. R. Sadeghpour, Phys. Rev. A 60, 3604 (1999).

[19] M. Aubert, N. Bessis, and G. Bessis, Phys. Rev. A 10, 51 (1974).

[20] C. W. McCurdy, D. A. Horner, and T. N. Rescigno, Phys. Rev. A 63, 022711 (2001).

[21] D. I. Abramov, A. Y. Kazakov, L. I. Ponomarev, S. Y. Slavyanov, and L. N. Somov, J. Phys. B 12, 1761 (1979).

[22] J. Rankin and W. R. Thorson, J. Comp. Phys. 32, 437 (1979).

[23] Y. S. Tergiman, Phys. Rev. A 48, 88 (1993).

[24] D. R. Bates and U. Öpik, J. Phys. B 1, 543 (1968).

[25] T. N. Rescigno, D. A. Horner, F. L. Yip, and C. W. McCurdy, Phys. Rev. A 72, 052709 (2005).

[26] M. Foster, J. Colgan, O. Al-Hagan, J. L. Peacher, D. H. Madison, and M. S. Pindzola, Phys. Rev. A 75, 062707 (2007).

[27] A. C. Wahl, P. E. Cade, and C. C. J. Roothaan, J. Chem. Phys. 41, 2578 (1964).

[28] E. L. Mehler and K. Ruedenberg, J. Chem. Phys 41, 2575 (1969).

[29] J. E. A. McCullough, J. Chem. Phys. 62, 3991 (1975).

[30] Y. V. Vanne and A. Saenz, J. Phys. B 37, 4101 (2004). 